\title{
A NOTE ON HOLOMORPHIC QUADRATIC DIFFERENTIALS ON THE UNIT DISK
}

\author{
GUOWU YaO
}

\begin{abstract}
Let $Q(\Delta)$ be the set of all integrable holomorphic quadratic differentials on the unit disk $\Delta$. The subset $Q_{0}(\Delta)$ of $Q(\Delta)$ is the set associated with $T_{0}$ classes in the universal Teichmüller space $T(\Delta)$. In this paper, it is shown that $Q_{0}(\Delta)$ is dense in $Q(\Delta)$. The infinitesimal version is also obtained.
\end{abstract}

\section{Introduction}

Let $\Delta$ be the unit disk in the complex plane $\mathbf{C}$. Denote by $\operatorname{Bel}(\Delta)$ the Banach space of Beltrami differentials $\mu=\mu(z) d \bar{z} / d z$ on $\Delta$ with finite $L^{\infty}$-norm and by $M(\Delta)$ the open unit ball in $\operatorname{Bel}(\Delta)$. Denoted by $Q(\Delta)$ the Banach space of the integrable holomorphic quadratic differentials on $\Delta$ with $L^{1}$-norm

$$
\|\varphi\|=\iint_{\Delta}|\varphi(z)| d x d y<\infty .
$$

In what follows, let $S Q(\Delta)$ denote the unit sphere and $Q_{1}(\Delta)$ the closed unit ball of $Q(\Delta)$.

For each element $\mu \in M(\Delta)$ there exists a uniquely determined quasiconformal mapping $f^{\mu}$ of $\Delta$ onto itself such that $f^{\mu}$ keeps $1,-1, i$ fixed and has the complex dilatation $\mu$. Two elements $\mu_{1}$ and $\mu_{2}$ are said to be Teichmüller equivalent (denoted by $\mu_{1} \sim \mu_{2}$ ) if and only if $\left.f^{\mu_{1}}\right|_{S^{1}}=\left.f^{\mu_{2}}\right|_{S^{1}}$. We denote the Teichmüller equivalence class of $\mu$ by $[\mu]$ or by $\left[f^{\mu}\right]$. The universal Teichmüller space $T(\Delta)$ is defined as the quotient space of $M(\Delta)$ under the equivalence relation. The point [0], the Teichmüller equivalence class of the trivial Beltrami differential $\mu=0$, is called the basepoint of $T(\Delta)$.

Define

$$
k_{0}([\mu]):=\inf \left\{\|v\|_{\infty}: v \in[\mu]\right\} .
$$

2010 Mathematics Subject Classification. Primary 30C62; Secondary 30F60.

Key words and phrases. Teichmüller space, quasiconformal mapping, quadratic differential, boundary dilatation.

The author was supported by the National Natural Science Foundation of China (Grant No. 11271216).

Received February 10, 2015; revised June 10, 2015. 
A NOTE ON HOLOMORPHIC QUADRATIC DIFFERENTIALS ON THE UNIT DISK

We say that $\mu$ is extremal in $[\mu]$ if $\|\mu\|_{\infty}=k_{0}([\mu]$ ) (the corresponding quasiconformal map $f^{\mu}$ is said to be extremal for its boundary values as well), uniquely extremal if $\|v\|_{\infty}>k_{0}(\mu)$ for any other $v \in[\mu]$.

As is well known, a Beltrami differential $\mu$ is extremal if and only if it has a so-called Hamilton sequence, namely, a sequence $\left\{\varphi_{n} \in S Q(\Delta)\right\}$, such that

$$
\lim _{n \rightarrow \infty} \iint \mu \varphi_{n}(z) d x d y=\|\mu\|_{\infty} .
$$

Define $h^{*}(\mu)$ to be the infimum over all compact subsets $E$ contained in $\Delta$ of the essential supremum norm of the Beltrami differential $\mu(z)$ as $z$ varies over $\Delta \backslash E$ and $h([\mu])$ to be the infimum of $h^{*}(v)$ taken over all representatives $v$ of the class $[\mu]$. It is obvious that $h([\mu]) \leq k_{0}([\mu])$. Following Earle and Li Zhong [4], $[\mu]$ is called a Strebel point if $h([\mu])<k_{0}([\mu])$; otherwise, $\tau$ is called a non-Strebel point. The result in [10] shows that the set of Strebel points is open and dense in $T(\Delta)$.

By the result in $[4][\mu]$ is a non-Strebel point if and only if the extremal in $[\mu]$ has a degenerating Hamilton sequence. A sequence in $Q(\Delta)$ is called degenerating if it converges to 0 uniformly on compact subsets of $\Delta$.

By Strebel's frame mapping criterion (see Chapter 4 in [6]), every Strebel point $[\mu]$ is represented by the uniquely-extremal Beltrami differential of the form $k \frac{\bar{\varphi}}{|\varphi|}$, where $k=k_{0}([\mu]) \in(0,1)$ and $\varphi$ is a unit vector in $Q(\Delta)$. Define the set of Strebel differentials $Q_{S}(\Delta)$ by

$$
Q_{S}(\Delta)=\left\{\varphi \in Q(\Delta) \backslash\{0\}: \exists k \in(0,1) \text { s.t. }\left[k \frac{\bar{\varphi}}{|\varphi|}\right] \text { is a Strebel point }\right\} .
$$

The complement $Q_{N}(\Delta)$ of $Q_{S}(\Delta)$ in $Q(\Delta)$ is called the set of non-Strebel differentials.

Define

$$
T_{0}(\Delta)=\{[\mu] \in T(\Delta): h([\mu])=0\} .
$$

$T_{0}(\Delta)$ is a closed subspace of $T(\Delta)$ [7] and every point in $T_{0}(\Delta) \backslash\{[0]\}$ is a Strebel point. The set $Q_{0}(\Delta)$ of $T_{0}$-class differentials is defined by

$$
Q_{0}(\Delta)=\left\{\varphi \in Q(\Delta) \backslash\{0\}: \exists k \in(0,1), \text { s.t. }\left[k \frac{\bar{\varphi}}{|\varphi|}\right] \in T_{0}(\Delta)\right\} .
$$

In [10], Lakic proved that both $Q_{S}(\Delta)$ and $Q_{N}(\Delta)$ are dense in $Q(\Delta)$. Naturally, we ask the following problem.

Problem 1. Is it true that $Q_{0}(\Delta)$ is dense in $Q(\Delta)$ ?

In this paper, we answer the problem affirmatively.

THeOrem 1. $Q_{0}(\Delta)$ is dense in $Q(\Delta)$. 
The theorem is proved in the next section. An infinitesimal version of Theorem 1 is obtained in Section 3.

\section{Proof of Theorem 1}

To prove Theorem 1, we need several lemmas.

The following lemma derives from the result in Lecture 4 of L. Bers' Courant Institute book [1].

Lemma 2.1. Let $\mu(z)$ be a $C^{n+\alpha}(0<\alpha<1, n=0,1,2, \ldots)$ function on a disk around zero, $\|\mu\|_{\infty}<1$. Let $a, b$ be two complex constants. Then any local solution $w=f(z)$ of the Beltrami equation

$$
w_{\bar{z}}=\mu(z) w_{z}, \quad w(0)=a, \quad w_{z}(0)=b,
$$

is of class $C^{n+1+\alpha}$.

Proof. By the theorem in Lecture 4 of L. Bers' Courant Institute book [1], the system (2.1) possesses, in the neighborhood of the origin, a solution $w(z)$ of class $C^{n+1+\alpha}$. Furthermore, by the similarity principle, any local solution $w=f(z)$ of the system $(2.1)$ is of class $C^{n+1+\alpha}$.

Let $C$ be a Jordan curve in $\mathbf{C}$. We say that $C$ is smooth if there is a parametrization $C: w(t), t \in[0,2 \pi]$ such that $w^{\prime}(t)$ is continuous and nowhere vanishing. The following result due to Kellogg can be found in [8] or [9] (Chapter X. Theorem 6, page 426).

LeMma 2.2. Let $\gamma$ map $\Delta$ conformally onto the inner domain of the Jordan curve $C$ of class $C^{1+\alpha}, 0<\alpha<1$. Then $\gamma^{\prime}$ and $\log \gamma^{\prime}$ are both $C^{\alpha}$ on $\bar{\Delta}$. In particular, $\gamma^{\prime}(z)$ is bounded from above and below on $\bar{\Delta}$.

A quasiconformal map $f: \Delta \rightarrow \Delta$ is called asymptotically conformal if for every $\varepsilon>0$ there is a compact set $E$ in $\Delta$ such that $f$ is $(1+\varepsilon)$-quasiconformal on $\Delta \backslash E$.

If $[\mu] \in T_{0}(\Delta)$, by Theorem 2 in Chapter 15 of [6] there is a so-called asymptotically extremal representative in $[\mu]$, say $\mu$, such that $f^{\mu}$ is asymptotically conformal. Conversely, it is clear that if $f^{\mu}$ is asymptotically conformal, then $h([\mu])=h^{*}(\mu)=0$ and $[\mu] \in T_{0}(\Delta)$.

Let $q: S^{1} \rightarrow S^{1}$ be a quasisymmetric map of the unit circle. The map $q$ is said to be symmetric (see [7], [13]) if

$$
\lim _{t \rightarrow 0} \frac{q\left(e^{i\left(t+t_{0}\right)}\right)-q\left(e^{i t_{0}}\right)}{q\left(e^{i t_{0}}\right)-q\left(e^{i\left(t_{0}-t\right)}\right)}=1,
$$

uniformly in $t_{0}$ for all points $z_{0}=e^{i t_{0}} \in S^{1}$. 
A NOTE ON HOLOMORPHIC QUADRATIC DIFFERENTIALS ON THE UNIT DISK 75

Strebel [13] and Fehlmann [5] showed respectively that a quasisymmetric map $q: S^{1} \rightarrow S^{1}$ is symmetric if and only if $q$ admits an asymptotically conformal extension to $\Delta$.

To sum up, we have the following conclusion.

LEMma 2.3. $[\mu] \in T_{0}(\Delta)$ if and only if the boundary map $q=\left.f^{\mu}\right|_{S^{1}}$ is symmetric.

Lemma 2.4. Suppose $\varphi$ belongs to $Q(\Delta)$ and is holomorphic in $\{z:|z|<1+\rho\}$ for some $\rho>0$. If $\varphi$ has no zero points on $S^{1}$, then $[\mu] \in T_{0}(\Delta)$ and $\varphi \in Q_{0}(\Delta)$ where

$$
\mu(z)=k \frac{\overline{\varphi(z)}}{|\varphi(z)|}, \quad k \in(0,1) .
$$

Proof. By the discreteness of zero points of non-zero holomorphic functions, there exists some small $\delta>0$ such that $\varphi$ has no zero points in $R=$ $\{z: 1-\delta<|z|<1+\delta\}$. Therefore, $\mu(z)$ is $C^{\infty}$ on $R$. Let $\tilde{f}$ be a quasiconformal mapping from $\{z:|z|<1+\delta\}$ onto the unit disk $\Delta$ with the complex dilatation $\mu$. Applying Lemma 2.1 on $R$ piecewise, we see that $\tilde{f}$ is $C^{\infty}$ on $R$. It yields that $C=\tilde{f}\left(S^{1}\right)$ is a smooth Jordan curve of class $C^{\infty}$.

Denote by $J$ the inner Jordan domain of $C$ and by $\gamma$ a conformal mapping from $\Delta$ onto $J$. By Carathéodory's theorem ([2] or see Theorem 2 on page 41 in [9]), $\gamma$ has a continuous extension to $\bar{\Delta}$. By Lemma 2.2, $\gamma^{\prime}(z)$ is nowhere vanishing on $\bar{\Delta}$. It derives that the inverse map $\gamma^{-1}$ is a $C^{1}$ diffeomorphism from $\bar{J}$ onto $\bar{\Delta}$.

Let $g=\gamma^{-1} \circ \tilde{f}$ be the quasiconformal mapping from $\Delta$ onto $\Delta$. It is clear that $g$ is at least $C^{1}$ on $\bar{\Delta}$ and hence $\left.g\right|_{S^{1}}$ is a symmetric map of $S^{1}$ by the definition. Notice that the Beltrami differential of $g$ is $\mu$. Therefore, by Lemma 2.3 we have $[\mu] \in T_{0}(\Delta)$.

Proof of Theorem 1. Given a holomorphic quadratic differential $\varphi$ in $Q(\Delta) \backslash\{0\}$, let $\varphi_{r}(z)=\varphi(r z)$ for $r \in(0,1)$. Obviously, $\varphi_{r}$ converges to $\varphi$ uniformly on compact subset of $\Delta$. Moreover, we have

$$
\lim _{r \rightarrow 1}\left\|\varphi_{r}-\varphi\right\|=0
$$

Since $\varphi$ is holomorphic in $\Delta$, the set of zero points of $\varphi$ is discrete in $\Delta$. We can choose a sequence $\left\{r_{n}\right\}$ in $(0,1)$ such that $r_{n} \rightarrow 1, n \rightarrow \infty$ and $\varphi_{n}\left(=\varphi_{r_{n}}\right)$ has no zero points on $S^{1}$.

Fix $k \in(0,1)$ and consider the Beltrami differential

$$
\mu_{n}(z)=k \frac{\overline{\varphi_{n}(z)}}{\left|\varphi_{n}(z)\right|} .
$$


Since $\varphi_{n}$ is holomorphic in $\left\{z:|z|<\frac{1}{r_{n}}\right\}$, it follows from Lemma 2.4 that $\left[\mu_{n}\right] \in T_{0}(\Delta)$ and hence $\varphi_{n} \in Q_{0}(\Delta)$. It is clear that

$$
\lim _{n \rightarrow \infty}\left\|\varphi_{n}-\varphi\right\|=0 \text {. }
$$

The proof of Theorem 1 is complete.

\section{Infinitesimal version of Theorem 1}

Two Beltrami differentials $\mu$ and $v$ in $\operatorname{Bel}(\Delta)$ are said to be infinitesimally equivalent if

$$
\iint_{\Delta} \mu \varphi d x d y=\iint_{\Delta} v \varphi d x d y, \quad \text { for any } \varphi \in Q(\Delta) .
$$

The tangent space $B(\Delta)$ of $T(\Delta)$ at the basepoint is defined as the set of the quotient space of $\operatorname{Bel}(\Delta)$ under the equivalence relation. Denote by $[\mu]_{B}$ the equivalence class of $\mu$ in $B(\Delta)$.

$B(\Delta)$ is a Banach space with its standard sup-norm

$$
\left\|[\mu]_{B}\right\|=\|\mu\|:=\sup _{\varphi \in S Q(\Delta)}\left|\iint_{\Delta} \mu \varphi d x d y\right|
$$

and infinitesimal metric

$$
\begin{aligned}
d\left([\mu]_{B},[v]_{B}\right) & :=\|\mu-v\| \\
& =\sup _{\varphi \in S Q(\Delta)}\left|\iint_{\Delta}(\mu-v) \varphi d x d y\right|, \quad[\mu]_{B},[v]_{B} \in B(\Delta) .
\end{aligned}
$$

Define the (infinitesimal) boundary dilatation $b\left([\mu]_{B}\right)$ of $[\mu]_{B}$ to be the infimum over all elements in the equivalence class $[\mu]_{B}$ of the quantity $b^{*}(v)$. Here $b^{*}(v)$ is the infimum over all compact subsets $E$ contained in $\Delta$ of the essential supremum of the Beltrami differential $v(z)$ as $z$ varies over $\Delta \backslash E$. We call $[\mu]_{B}$ in $B(\Delta)$ an infinitesimal Strebel point if $b\left([\mu]_{B}\right)<\|\mu\|$. Clearly, the basepoint is not a Strebel point since $b\left([0]_{B}\right)=\left\|[0]_{B}\right\|=0$. It follows from Reich's infinitesimal frame mapping theorem (see Theorem 2.4 in [11]) that if $[\mu]_{B}$ is an infinitesimal Strebel point, then there exists a unique vector $\varphi$ in $Q(\Delta)$ such that $\mu$ and $\|\mu\| \frac{\bar{\varphi}}{|\varphi|}$ are infinitesimally equivalent.

In [3], the semi-norm $\beta$ on $B(\Delta)$ is defined by

$$
\beta(\mu)=\sup _{\varphi_{n}} \limsup _{n \rightarrow \infty}\left|\iint_{\Delta} \mu \varphi_{n} d x d y\right|,
$$

where the supremum is taken over all degenerating sequences in $Q_{1}(\Delta)$. It is also proved in [3] that $\beta(\mu)=b\left([\mu]_{B}\right)$. 
Define

$$
B_{0}(\Delta)=\left\{[\mu]_{B} \in B(\Delta): b\left([\mu]_{B}\right)=0\right\} .
$$

Then $B_{0}(\Delta)$ is a linear subspace of $B(\Delta)$ and every point in $B_{0}(\Delta) \backslash\left\{[0]_{B}\right\}$ is an infinitesimal Strebel point.

In a parallel way, one can define the sets of infinitesimal Strebel and nonStrebel differentials respectively. Whereas there is no difference between these two kinds of Strebel (or non-Strebel) differentials due to the property that a(n) (infinitesimal) Strebel point has no degenerating Hamilton sequence and vice versa. So, we still use $Q_{N}(\Delta)$ and $Q_{S}(\Delta)$ to denote them.

By use of a trick in [10] for the infinitesimal setting, one easily proves (also see [12]) that the set of infinitesimal Strebel points is open and dense in $B(\Delta)$.

We now define a new set of $B_{0}$-class (parallel to $T_{0}$-class) differentials independently. The set $Q_{Z}(\Delta)$ of $B_{0}$-class differentials is defined by

$$
Q_{Z}(\Delta)=\left\{\varphi \in Q(\Delta) \backslash\{0\}: \exists k \in \mathbf{R} \text {, s.t. }\left[k \frac{\bar{\varphi}}{|\varphi|}\right]_{B} \in B_{0}(\Delta)\right\} .
$$

The infinitesimal counterpart of Theorem 1 is as follows.

THeOrem 2. $Q_{Z}(\Delta)$ is dense in $Q(\Delta)$.

Proof. Let $\varphi$ be a holomorphic quadratic differential in $Q(\Delta) \backslash\{0\}$. Use the same denotation as in the proof of Theorem 1. Since $\mu_{n}$ is continuous on some ring domain $U_{n}=\left\{z: \rho_{n} \leq|z| \leq 1\right\}, \rho_{n} \in(0,1)$, where $\mu_{n}$ is defined by (2.3). It follows from the following Lemma 3.1 that $\left[\mu_{n}\right]_{B}$ belongs to $B_{0}(\Delta)$ and hence $\varphi_{n} \in Q_{Z}(\Delta)$. It yields directly that $Q_{Z}(\Delta)$ is dense in $Q(\Delta)$.

Lemma 3.1. Suppose $\mu \in \operatorname{Bel}(\Delta)$ is continuous in the ring domain $\{z: \rho \leq|z| \leq 1\}, \rho \in(0,1)$. Then $[\mu]_{B} \in B_{0}(\Delta)$.

Proof. Since $b\left([\mu]_{B}\right)=\beta(\mu)$, it suffices to prove that $\beta(\mu)=0$. For any given degenerating sequence $\left\{\psi_{n}\right\}$ in $S Q(\Delta)$, we need to prove that

$$
\limsup _{n \rightarrow \infty}\left|\iint_{\Delta} \mu \psi_{n} d x d y\right|=0 \text {. }
$$

Let $\epsilon>0$ and

$$
P(z, \bar{z})=\sum_{k, l=0}^{N} c_{k l} z^{k} \bar{z}^{l}=\sum_{k, l=0}^{N} c_{k l} r^{k+l} e^{i(k-l) \theta}
$$

be a polynomial in $z$ and $\bar{z}$ for which

$$
|\mu(z)-P(z, \bar{z})| \leq \epsilon, \quad \rho \leq|z| \leq 1 .
$$


Notice that

$$
\int_{0}^{2 \pi} e^{i n \theta} d \theta=0, \quad n=1,2, \ldots
$$

We have

$$
\begin{aligned}
\iint_{\rho \leq|z| \leq 1} P(z, \bar{z}) \psi_{n}(z) d x d y & =\iint_{\rho \leq|z| \leq 1}\left[\sum_{k, l=0}^{N} c_{k l} z^{k} \bar{z}^{l} \sum_{m=0}^{\infty} \frac{\psi_{n}^{(m)}(0)}{m !} z^{m}\right] d x d y \\
& =\int_{0}^{2 \pi} \int_{\rho}^{1}\left[\sum_{k, l=0}^{N} \sum_{m=0}^{\infty} c_{k l} \frac{\psi_{n}^{(m)}(0)}{m !} r^{m+k+l+1} e^{i(m+k-l) \theta}\right] d r d \theta \\
& =\int_{0}^{2 \pi} \int_{\rho}^{1}\left[\sum_{k, l=0}^{N} \sum_{m=0}^{N} c_{k l} \frac{\psi_{n}^{(m)}(0)}{m !} r^{m+k+l+1} e^{i(m+k-l) \theta}\right] d r d \theta \\
& =\sum_{m=0}^{N} \frac{\psi_{n}^{(m)}(0)}{m !} \sum_{k, l=0}^{N} c_{k l} \int_{\rho}^{1} r^{m+k+l+1} d r \int_{0}^{2 \pi} e^{i(m+k-l) \theta} d \theta
\end{aligned}
$$

Since $\psi_{n}$ converges to 0 uniformly on compact subsets of $\Delta$, it holds that

$$
\lim _{n \rightarrow \infty} \psi_{n}^{(m)}(0)=0
$$

for any fixed $m$. Therefore,

$$
\lim _{n \rightarrow \infty} \iint_{\rho \leq|z| \leq 1} P(z, \bar{z}) \psi_{n}(z) d x d y=0 .
$$

It follows readily that

$$
\lim _{n \rightarrow \infty}\left|\iint_{\Delta} \mu(z) \psi_{n}(z) d x d y\right|=\left|\lim _{n \rightarrow \infty} \iint_{\rho \leq|z| \leq 1}(\mu(z)-P(z, \bar{z})) \psi_{n}(z) d x d y\right| \leq \epsilon .
$$

Thus, we obtain (3.1).

Lemma 3.1 indicates that a Beltrami differential belongs to $B_{0}(\Delta)$ if it is continuous near the boundary $S^{1}$. It is unclear whether there is a similar conclusion for the $T_{0}$ classes. Precisely, we pose the following problem.

Problem 2. Suppose $\mu$ is continuous in the ring domain $\{z: \rho \leq|z| \leq 1\}$, $\rho \in(0,1)$. Can we say that $[\mu] \in T_{0}(\Delta)$ ?

The author believes that the boundary map $\left.f^{\mu}\right|_{S^{1}}$ corresponding to $\mu$ is Lipschitz or even bi-Lipschitz if $\mu$ is continuous near $S^{1}$.

At last, we note that it is very interesting to determine the relation between the two sets $Q_{0}(\Delta)$ and $Q_{Z}(\Delta)$. The following problem is open. 
Problem 3. Is it true that $Q_{0}(\Delta)$ and $Q_{Z}(\Delta)$ coincide?

Acknowledgements. The author would like to thank the referee for his comments and suggestions which improved the exposition and clarity.

\section{REFERENCES}

[1] L. Bers, Riemann surfaces, Courant institute notes, New York University Press, 1957-1958.

[2] C. CarathÉodory, Über die Begrenzung einfach zusammenhängender Gebiete, Math. Ann. 73 (1913), 323-370 (in German).

[3] C. J. Earle, F. P. Gardiner and N. Lakic, Asymptotic Teichmüller space, Part I: The complex stucture, Contemp. Math. 256, Amer. Math. Soc. Providence, RI, 2000, 17-38.

[ 4 ] C. J. EARLE AND Z. LI, Isometrically embedded polydisks in infinite-dimensional Teichmüller spaces, J. Geom. Anal. 9 (1999), 51-71.

[5] R. Fehlmann, Ueber extremale quasikonforme Abbildungen, Comment. Math. Helv. 56 (1981), 558-580 (in German).

[6] F. P. Gardiner and N. LaKic, Quasiconformal Teichmüller theory, Amer. Math. Soc. Providence, RI, 2000.

[7] F. P. Gardiner and D. P. Sullivan, Symmetric structures on a closed curve, Amer. J. Math. 114 (1992), 683-736.

[8] G. M. Goluzin, Geometric function theory, Nauka, Moscow, 1966 (in Russian).

[9] G. M. Goluzin, Geometric theory of function of a complex variable, Translations of mathematical monographs 26, Amer. Math. Soc. 1969.

[10] N. LaKic, Strebel points, Contemp. Math. 211, Amer. Math. Soc. Providence, RI, 1997, 417-431.

[11] E. ReICH, An extremum problem for analytic functions with area norm, Ann. Acad. Sci. Fenn. Ser. A. I. Math. 2 (1976), 429-445.

[12] Y. Shen AND X. Liu, Some remarks on holomorphic quadratic differentials, Adv. Math. 33 (2004), 471-476 (in Chinese).

[13] K. Strebel, On the existence of extremal Teichmüller mappings, J. Anal. Math. 30 (1976), 464-480.

Guowu Yao

Department of Mathematical Sciences

TSINGHUA UNIVERSITY

BEIJING, 100084

P. R. CHINA

E-mail: gwyao@math.tsinghua.edu.cn 